\title{
Novel Single-Molecule Technique by Single-Object Scattering Sampling (SOSS)
}

\author{
Hyotcherl Ihee \\ Center for Time-Resolved Diffraction, Department of Chemistry, KAIST, Daejeon 305-701, Korea \\ ${ }^{*}$ E-mail: hyotcherl.ihee@kaist.ac.kr \\ Received April 26, 2011, Accepted May 22, 2011
}

Key Words : Single molecule, Protein structural dynamics, Transition state, Molecular fluctuation dynamics, Wave function

Here we propose a new single-molecule experiment that can directly measure the conformation fluctuations of a single object and detect the structure of rarely populated states. This experiment is performed by sampling random scattering snapshots of single objects using ultrashort, ultraintense, coherent X-ray pulses (Figure 1). If the X-ray pulse is shorter than the time scale of the motion of an object and intense enough to generate a useful X-ray scattering image from a single object, the measured scattering image contains the structural information on the single object as if its motion is frozen in time. If one structural parameter of interest can be extracted from the single-object scattering image, each image yields a structure parameter value corresponding to the conformation of the single object at the instant of the interaction with the X-ray pulse. By collecting a large number of such images, many conformations of the single object are effectively sampled and a distribution of the structural para-

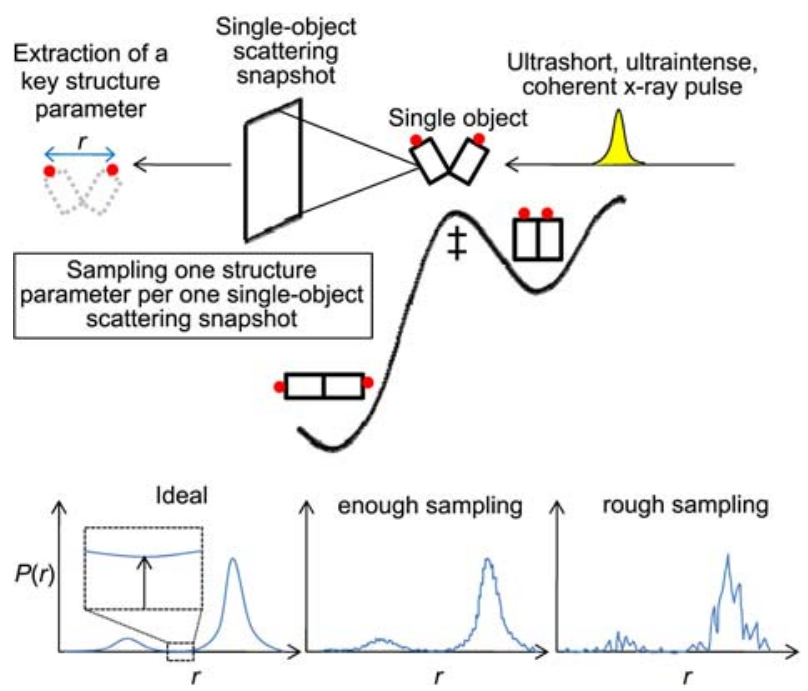

Figure 1. Concept of Single-Object Scattering Sampling (SOSS). A single-shot single-molecule X-ray scattering pattern captures the structure information at the instant of the interaction of the X-ray pulse and the molecule and has potential to sample even the structure of a short-lived transition state (shown in the figure) or a rarely populated state. Collecting a large number of the singlemolecule scattering patterns can sample the entire conformation space, $P(r)$, of the molecule with respect to the key structure parameter $r$, the distance between two heavy scatterers (red dots). The ideal $P(r)$ that has finite probability (indicated as an arrow) even at the transition state can be approached with enough sampling. meter, $P(r)$, can be extracted. In addition, this technique opens the possibility of experimentally capturing the structure of a rarely populated structure that has short lifetime and low population (for example, a short-lived transition state of a small molecule or a functionally important but rarely populated conformation of a biomolecule). X-ray free electron lasers (XFEL) can provide coherent femtosecond X-ray pulses containing $10^{13}$ photons per pulse that can be potentially focused to $10 \mathrm{~nm}$ or even smaller size. In fact, this idea can also be applied to other scattering particles such as electrons as long as coherent, ultrashort and ultraintense pulses of the particles become available. Here we display the conceptual foundation and the potential impact of this idea, which we name single-object scattering sampling (SOSS), building on our previous description. ${ }^{1}$ We use the term "object" instead of "molecule" because this technique has the potential to be applied to any objects including small molecules, proteins, nucleic acids, macromolecular complexes, nano-sized systems, aggregates and micro-sized objects. In this paper, we use small molecules and biomolecules as potential application examples.

Nuclear vibration of a simple diatomic molecule, for example $I_{2}$, can be described in the framework of nuclear vibrational wave functions, but the shape of the vibrational wave function is usually assumed without experimental verification. Imagine an ultrashort, ultraintense, coherent Xray pulse is focused to a single $\mathrm{I}_{2}$ molecule. The single molecule would be eventually destroyed by Coulomb explosion of highly charged nuclei generated by X-ray-induced ionization, but its scattering pattern still contains the structural information before radiation damage destroys the single molecule as far as the X-ray pulse is short enough. As far as the X-ray pulse width is shorter than a period of the vibrational motion, the scattering pattern can capture one instance during the vibration. Then, by measuring a large number of single-molecule scattering patterns, we can sample the entire distribution of I-I distance, which corresponds to the square of the wave function, i.e. the probability, of the ground vibrational state. By combining SOSS with vibrational excitation, we can also determine the shape of the squared wave function of a vibrationally excited state.

We can expand this idea in the context of reaction. The transition state connecting the reactant to the product is elusive due to its extremely short lifetime and low popula- 
tion probability. For a simple polyatomic molecule, for example $\mathrm{C}_{2} \mathrm{H}_{4} \mathrm{I}_{2}$ (1,2-Diiodoethane), the stable anti and gauche isomers convert by rotation around the $\mathrm{C}-\mathrm{C}$ bond through rotational transition states. The molecular structures of the anti and gauche isomers can be determined via various experimental techniques, but those of the rotational transition states have never been directly captured. In ensemble measurements, even with infinitely high signal-tonoise ratio, the signal from highly populated structures (in this case the anti and gauche isomers) always dominates, obscuring the signature of rarely populated structures (in this case the transition states). In contrast, single-molecule spectroscopy probes one molecule at a time and thus in principle has the potential of capturing the rarely populated structure without being obscured by the signal from dominant species at the single-molecule level, but typical single molecule spectroscopy using fluorescence or absorption at optical and IR wavelengths does not provide temporal and spatial resolutions required for the observation of the short-lived structure. The SOSS can remedy this situation because X-ray scattering (diffraction) provides higher spatial resolution than optical or IR absorption (or fluorescence) and ultrashort X-ray pulses available at XFEL provides the time resolution as short as a period of molecular vibration and internal rotation. Since the scattering intensity from a single $\mathrm{C}_{2} \mathrm{H}_{4} \mathrm{I}_{2}$ molecule mainly arises from the interference of heavy atoms, that is two iodine atoms, this system can be considered as an elongated $\mathrm{I}_{2}$ molecule. A distribution of I-I distance can be obtained from many singlemolecule images and converted to a distribution of dihedral angle, which is more relevant for describing the rotational motion. As in $\mathrm{I}_{2}$, this distribution corresponds to the square of the rotational wave function. With a large number of sampling, there exists finite probability to capture the structure of a single $\mathrm{C}_{2} \mathrm{H}_{4} \mathrm{I}_{2}$ molecule at or near the rotational transition state. In addition, rotational excitation should help the detection of transition states as the excitation of higher rotational states increases the probability for population of the rotational transition states.

The concept of SOSS can be applied to biomolecules as well. The widespread idea of single-molecule biomolecular imaging using X-ray diffraction focuses on determining the atomic structures of a protein, of which three-dimensional structure is not known, by averaging a significant number of single-shot diffraction patterns. ${ }^{2}$ In contrast, SOSS is designed to map out the unknown conformational space of a protein, whose static structure may be already available, by extracting various conformations of the protein from many single-shot scattering patterns. In other words, we aim to explore the conformation fluctuations of proteins of known structure rather than determine the average molecular structure itself. One major concern is that the structural complexity of biomolecules and low signal-to-noise ratio may not allow the extraction of the structural parameters of interest from a single-shot, single-molecule X-ray diffraction image. To alleviate this issue, we can attach two or more heavy scattering probes, for example gold nanocrystals, to specific locations in the biomolecule via mutagenesis and chemical coupling so that the scattering signal is dominated by the contribution of the heavy scattering probes. In other words, we enhance the contribution from a specific structure parameter (in this case, the distance between the two probes), while opting to discard all the detailed structural information contained in the single-shot, single-molecule diffraction patterns. In this manner, we can increase the signal-to-noise ratio of a single-molecule, single-shot scattering image while simplifying the structural information of the protein.

As an example of applying SOSS to biomolecules, we can imagine a protein in the equilibrium between the folded and unfolded states or an RNA possessing multiple conformations. ${ }^{3}$ When two probes are attached to specific locations in the protein, the distribution of the distance between the probes can be simulated by molecular dynamics simulations, but the exact shape of such distribution has never been measured, not even by widespread single-molecule spectroscopy. Single-molecule FRET measurement using two dye labels has limitations in spatial and time resolutions and often renders the complex distribution of conformation into simplified two or multiple states, so-called "FRET-on" and "FRET-off" states. Although FRET efficiency can be translated into the distance information, its temporal resolution may blur the ideal $P(r)$, even with enough signal to noise ratio. By contrast, SOSS can directly determine the distance between the two probes with higher spatial resolution without blurring caused by low time resolution. Therefore, from the occurrence distribution of the distance obtained from SOSS, the entire conformational space of the folding-unfolding pathway can be mapped out. In other words, the distribution of structure can be visualized in details thanks to the time and spatial resolution of SOSS.

Experimental realization of SOSS would have to overcome many obstacles. The most important issue for the success of SOSS is whether a sufficient number of X-ray photons can be detected so that a structure parameter can be extracted from a single image of good signal-to-noise ratio. Detailed calculations of SOSS signals are in progress and preliminary results show that the small molecules such as $\mathrm{I}_{2}$ and $\mathrm{C}_{2} \mathrm{H}_{4} \mathrm{I}_{2}$ may be beyond the technical capability of the current X-ray free electron lasers unless extremely tight focusing of X-ray light down to $1 \mathrm{~nm}$ can be achieved. On the other hand, biomolecules labeled with two gold nanoparticles are expected to greatly enhance the number of scattered X-ray photons and experimental realization is within reach with the current X-ray free electron laser sources.

Acknowledgments. This work was supported by the Creative Research Initiatives (Center for Time-Resolved Diffraction) of MEST/NRF.

\section{References}

1. Kim, J.; Kim, K. H.; Lee, J. H.; Ihee, H. Acta Crystallogr A 2010 , $66,270$.

2. Neutze, R.; Wouts, R.; van der Spoel, D.; Weckert, E.; Hajdu, J. Nature 2000, 406, 752 .

3. Hong, H.; Kim, Y. G.; Hohng, S. B Kor Chem Soc 2010, 31, 1021. 